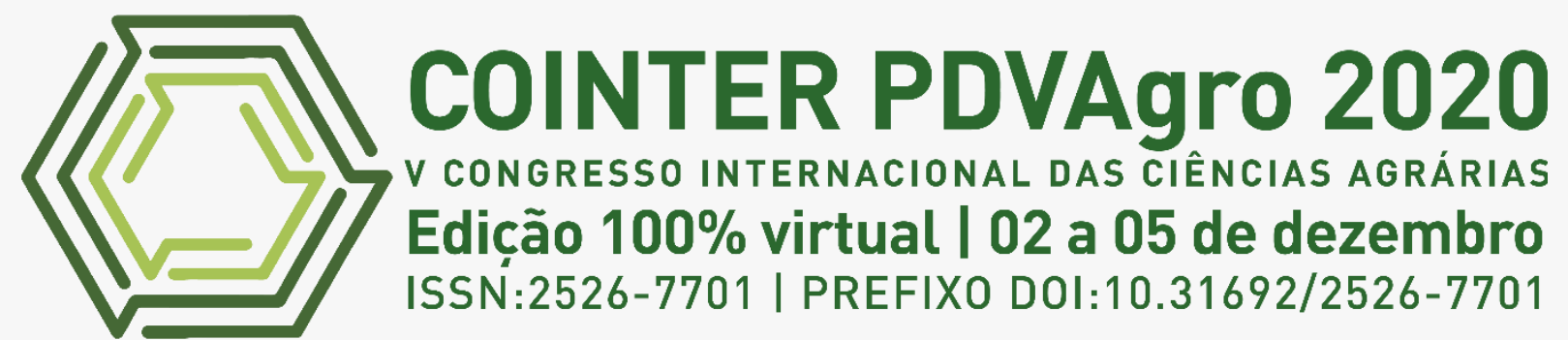

\title{
AVALIAÇÃO DA EXTRAÇÃO, COMÉRCIO E BENEFICIAMENTO DE MADEIRA EM TORA NO PERÍODO DE 2006 A 2016 NA MESORREGIÃO SUDOESTE PARAENSE, BRASIL.
}

\section{EVALUACIÓN DE LA EXTRACCIÓN, EL COMERCIO Y EL PROCESAMIENTO DE MADEIRA EN EL PERÍODO 2006-2016 EN LA MESORREGIÓN SUROESTE DE PARÁ, BRAZIL.}

\section{EVALUATION OF THE EXTRACTION, TRADE AND PROCESSING OF TIMBER IN THE PERIOD FROM 2006 TO 2016 IN THE SOUTHWEST MESOREGION OF PARÁ, BRAZIL.}

\author{
Apresentação: Comunicação Oral
}

Camila da Silva Damasceno ${ }^{1}$; Stefany de Andrade Silva ${ }^{2}$; Igor Cristian de Oliveira Vieira ${ }^{3}$ Elesandra da Silva Araújo $^{4}$

\section{RESUMO}

DOI: https://doi.org/10.31692/2526-7701.VCOINTERPDVAgro.0313

A Amazônia é um dos polos madeireiros que contribui para a economia do Brasil, onde a mesorregião do sudoeste paraense possui um grande destaque para a extração, comercialização e beneficiamento de madeira, por este motivo o presente trabalho tem como finalidade a análise da extração, comércio e beneficiamento de tora de madeira nativa na mesorregião Sudoeste Paraense no período de 2006 a 2016. Os dados foram obtidos através dos relatórios da Secretária de Estados de Meio Ambiente e Sustentabilidade - SEMAS, referente à Extração e Comércio de Toras de Madeira Nativa por Município que apresenta os valores de extração de madeira em tora de volume $\left(\mathrm{m}^{3}\right)$ e o valor correspondente (R\$), também foi utilizado o relatório de Beneficiamento e Comércio de Produtos de Madeira por Espécies Florestais obtido pelo Sistema de Comercialização e Transporte de Produtos Florestais (SISFLORA), que apresenta os produtos beneficiados, o volume $\left(\mathrm{m}^{3}\right)$ e seu respectivo valor $(\mathrm{R} \$)$, ambos durante o período consolidado de 2006 à 2016. Os resultados apresentaram que no parâmetro de extração e comércio por espécie, o Ipê como é vulgarmente conhecido com maior receita (R\$) e volume $\left(\mathrm{m}^{3}\right)$ em sete municípios Rurópolis, Trairão, Uruará, Senador José Porfírio, Itaituba, Altamira e Anapu, e o beneficiamento foi verificado em quatro tipos: aproveitamentos da madeira, madeira serrada, decking e toras de madeira nativa. O município de Uruará foi o destaque por extrair o Ipê, tendo produzido 72167, 84 vol. $\mathrm{m}^{3}$ gerando R $\$ 27.338 .405,02$. A espécie madeireira Tabebuia serratifolia (Vahl) Nichols teve o maior valor em vol. $\mathrm{m}^{3}$ entre os produtos de beneficiamento analisados, entre os produtos a madeira serrada e o decking tiveram o maior volume $\left(\mathrm{m}^{3}\right)$ e na receita $(\mathrm{R} \$)$, totalizando entre todos os produtos o volume de $9997.429,142 \mathrm{~m}^{3}$ e a receita em $\mathrm{R} \$ 2.310 .655 .236,27$. A segunda maior espécie em extração e comercialização é a Maçaranduba nos municípios de Brasil Novo, Aveiro e Pacajá totalizando em volume 221.451,506 $\mathrm{m}^{3}$ de madeira e a receita em $\mathrm{R} \$ 40.846 .099,3$ possuindo destaque no beneficiamento em toras de madeira nativa e decking, totalizando entre todos os produtos um volume

\footnotetext{
${ }^{1}$ Engenharia Florestal, Universidade Federal Rural da Amazônia, camilasdamasceno00@ gmail.com

${ }^{2}$ Engenharia Florestal, Universidade Federal Rural da Amazônia, stef.engflorestal@ gmail.com

${ }^{3}$ Mestrando em Agronomia, Universidade Estadual Paulista, Jaboticabal, Jaboticabal/SP, igor.vieira@unesp.br

${ }^{4}$ Doutoranda em Engenharia de Biomateriais, Universidade Federal de Lavras, elesandra.araujo@hotmail.com
} 
de $1.573 .177,19 \mathrm{~m}^{3}$ e o valor de $\mathrm{R} \$ 709.864 .339,84$. Entre os municípios que compõem a microrregião, Pacajá é o mais importante na extração de toras, a soma total da sua receita é R $\$ 230.522 .780,47$ e de volume 1.657.410, $11 \mathrm{~m}^{3}$ total. Esse estudo feito com as análises dos dados permite compreender a importância da dinâmica da indústria madeireira da floresta Amazônica no Pará.

Palavras-Chave: Amazônia; Economia florestal; Polos madeireiros;

\section{RESUMEN}

La Amazonía es uno de los postes de madera que contribuye a la economía de Brasil, donde la mesorregión del suroeste de Pará tiene un gran énfasis en la extracción, comercialización y procesamiento de madera, por esta razón el presente trabajo tiene como objetivo el análisis de la extracción, el comercio y procesamiento de troncos de madera nativa en la mesorregión Suroeste Paraense de 2006 a 2016. Los datos se obtuvieron a través de informes de la Secretaría de Estado de Ambiente y Sustentabilidad - SEMAS, referidos a la Extracción y Comercio de Troncos de Madera Nativa por Municipio que presenta los valores de extracción de madera en volumen $\left(\mathrm{m}^{3}\right)$ y el valor correspondiente ( $\mathrm{R}$ \$), el informe de Beneficio y Comercio de Productos de Madera por Especies Forestales obtenido por el Sistema de Comercialización y Transporte de Productos Forestales ( SISFLORA), que presenta los productos beneficiados, el volumen $\left(\mathrm{m}^{3}\right)$ y su respectivo valor $(\mathrm{R} \$)$, ambos durante el período de consolidación de 2006 a 2016. Los resultados mostraron que en el parámetro de extracción y comercio por especies, Ipê como se le conoce comúnmente con mayor ingreso (R \$) y volumen $\left(\mathrm{m}^{3}\right)$ en siete municipios Rurópolis, Trairão, Uruará, Senador José Porfírio, Itaituba, Altamira y Anapu, y se verificó el procesamiento en cuatro tipos: uso de madera, madera aserrada, entablado y troncos de madera nativa. El municipio de Uruará fue el destacado por la extracción del Ipê, habiendo producido 72167, 84 vol. $\mathrm{m}^{3}$ generando R \$27.338.405,02. La especie maderable Tabebuia serratifolia (Vahl) Nichols tuvo el mayor valor en vol. $\mathrm{m}^{3}$ entre los productos procesados analizados, entre los productos madera aserrada y entablado tuvo el mayor volumen $\left(\mathrm{m}^{3}\right)$ y en ingresos $(\mathrm{R} \$)$, totalizando entre todos los productos tuvieron un volumen de $9997.429 .142 \mathrm{~m}^{3}$ y un ingreso de $\mathrm{R} \$$ 2.310.655.236,27. La segunda especie más importante en extracción y comercialización es Maçaranduba en los municipios de Brasil Novo, Aveiro y Pacajá, con un volumen total de 221.451,506 $\mathrm{m}^{3}$ de madera e ingresos de R \$40.846.099,3 con énfasis en el procesamiento de troncos y tarimas de madera nativa. , totalizando entre todos los productos un volumen de $1.573 .177,19 \mathrm{~m}^{3}$ y el valor de $\mathrm{R} \$ 709.864 .339,84$. Entre los municipios que conforman la microrregión, Pacajá es el más importante en la extracción de troncos, la suma total de sus ingresos es de R \$230.522.78047 y el volumen $1.657 .410,11 \mathrm{~m}^{3}$ en total. Este estudio realizado con análisis de datos nos permite comprender la importancia de la dinámica de la industria maderera en la selva amazónica de Pará.

\section{Palabras Clave:}

\section{ABSTRACT}

The Amazon is one of the logging hubs that contributes to the economy of Brazil, where the mesoregion of the southwest of Pará has a great emphasis on the extraction, commercialization and processing of wood, for this reason the present work aims to analyze extraction, trade and processing of native wood logs in the Southwest Paraense mesoregion from 2006 to 2016. The data were obtained through reports from the Secretary of State for Environment and Sustainability - SEMAS, referring to the Extraction and Trade of Logs of Native Wood by Municipality that presents the values of timber extraction in volume $\left(\mathrm{m}^{3}\right)$ and the corresponding value $(\mathrm{R} \$)$, the report of Beneficiation and Trade in Wood Products by Forest Species obtained by the System for Commercialization and Transport of Forest Products ( SISFLORA), which presents the benefited products, the volume $\left(\mathrm{m}^{3}\right)$ and their respective value ( $\mathrm{R} \$$ ), both during the consolidation period from 2006 to 2016. The results showed that in the parameter of extraction and trade by species, Ipê as it is commonly known with higher revenue $(\mathrm{R} \$)$ and volume $\left(\mathrm{m}^{3}\right)$ in seven municipalities Rurópolis, Trairão, Uruará, Senador José Porfírio, Itaituba, Altamira and Anapu, and the processing was verified in four types: wood use, sawn wood, decking and native wood logs. The municipality of Uruará was the highlight for extracting the Ipê, having produced 72167, 84 vol. $\mathrm{m}^{3}$ generating R \$27,338,405.02. The timber species Tabebuia serratifolia (Vahl) Nichols had the highest value in vol. $\mathrm{m}^{3}$ among the processed products analyzed, among the products sawn wood and decking had the highest volume $\left(\mathrm{m}^{3}\right)$ and in revenue $(\mathrm{R} \$)$, totaling between all products had a volume of $9997,429,142 \mathrm{~m}^{3}$ and revenue of $\mathrm{R} \$ 2,310,655,236.27$. The second largest species in extraction and 
commercialization is Maçaranduba in the municipalities of Brasil Novo, Aveiro and Pacajá, with a total volume of $221,451.506 \mathrm{~m}^{3}$ of wood and revenue of $\mathrm{R} \$ 40,846,099.3$ with emphasis on the processing of native wood logs and decking, totaling among all products a volume of $1,573,177.19 \mathrm{~m}^{3}$ and the value of $\mathrm{R} \$ 709,864,339.84$. Among the municipalities that make up the microregion, Pacajá is the most important in the extraction of logs, the total sum of its revenue is $\mathrm{R} \$ 230,522,780,47$ and volume $1,657,410,11 \mathrm{~m}^{3}$ total. This study carried out with data analysis allows us to understand the importance of the dynamics of the timber industry in the Amazon rainforest in Pará.

Keywords:

\section{INTRODUÇÃO}

A indústria madeireira é um dos setores considerado importante em escala mundial por ser um setor da economia brasileira de grande representatividade e ter grande participação no PIB do país, além da geração de rendas, tributos, divisas e empregos. (NUNES; MELO; TEIXEIRA, 2012).

A região amazônica possui um grande potencial para a produção de madeira, além de ser habitat de vasta biodiversidade animal e vegetal (MINISTÉRIO DO MEIO AMBIENTE, 2016). Essa região desempenha um importante papel no cenário industrial na extração, comércio e beneficiamento de madeira devido sua biodiversidade de espécies florestais, sendo considerado um dos principais pilares econômicos de madeira nativa no Brasil tornando-se relevante para o mercado interno e externo na Amazônia (IMAZON, 2010).

A atividade madeireira é considerado um importante propulsor para a economia, resultando na geração de empregos e receitas de forma direta e indireta, e se apresentando como fonte legal e renovável na Amazônia, com a adoção de uma extração seletiva (ASNER et al., 2009) respeitando o períodos ideias para o corte, e manutenção de espécies maduras reservadas para a produção de sementes, ajudando na regeneração e estrutura da floresta em pé (DING et al., 2017).

A indústria de extração e processamento de madeira encontra-se em lugar de destaque na economia na Amazônia, somada as atividades de mineração e agropecuária. Este setor extrai aproximadamente 24,5 milhões de metros cúbicos de madeira em tora, o equivalente a cerca de 6,2 milhões de árvores por ano. Tal matéria prima gera ainda mais receitas com o seu processamento que gera em torno de 10,4 milhões de metros cúbicos de madeira processada, que resultam em (tábuas, produtos beneficiados, laminados, compensados e outros.) (LENTINI et al., 2006).

O Pará encontra-se dividido em seis Mesorregiões que são Baixo Amazonas Paraense, Marajó, Metropolitana de Belém, Nordeste Paraense, Sudoeste Paraense e Sudeste Paraense, nas quais o Sudeste, Sudoeste e Nordeste paraense são os pioneiros na exploração e comercialização de madeira legal e ilegal, no entanto, possa atividade ter caráter predatório a 
área florestal em 2002 teve 232 mil hectares de floresta certificadas (VERÍSSIMO, 2002).

O sudoeste paraense foi a segunda mesorregião no ano de 2015 que apresentou o maior destaque na comercialização e extração em tora com $20 \%$ ficando atrás do baixo amazonas com 44\%, os municípios do sudoeste paraense com a maior evidência nesse percentual é Aveiro, Novo progresso e Uruará (LUZ, 2015).

Nesse contexto, o estudo tem como objetivo avaliar o cenário da extração, comércio e beneficiamento de madeira em tora no período de 2006 a 2016 da mesorregião Sudoeste Paraense. Apesar do sistema não ter os dados atualizados, ainda sim é possível fazer a avaliação pretendida.

\section{FUNDAMENTAÇÃO TEÓRICA}

A atividade madeireira esteve restrita por muitos anos às florestas de várzea que eram localizadas na beira dos rios da Amazônia, nessa época a extração de madeira era bastante seletiva e tinha poucos impactos. Essa exploração começou a se intensificar com a construção das rodovias BR-010 e BR-230, e o polo madeireiro começou a se concentrar nessas áreas e tornou-se uma grande potência na economia da região. (IMAZON, 2010).

Com o desenvolvimento da região foram instaladas inúmeras empresas do setor madeireiro ao redor das rodovias, os polos foram se aglomerando nos centros que ofereciam serviços de infraestruturas (energia, comunicação, saúde e sistemas bancários) e mão-de-obra disponível. Uma localidade é considerada um polo madeireiro quando o volume de sua extração e consumo anual de madeira em tora é igual ou superior a 100 mil metros cúbicos. (IMAZON, 2010)

Devido à dificuldade de obter matéria prima florestal, se observa uma grande extração madeireira nas fronteiras da região Amazônica em razão das indústrias madeireiras estarem interligadas com a diversidade de espécies madeireiras (PAIVA, 2009). O início do processo de degradação se dá devido a extração da madeira em uma área sendo explorado primeiramente as árvores com maior valor econômico e maior volume e subsequentemente explorando espécies importantes para a regeneração das florestas. (MONTEIRO et al, 2004).

A extração de toras de madeira e o beneficiamento no Estado do Pará onde está a maior parte da floresta nativa, é um dos destaques nas atividades madeireiras possuindo $51 \%$ de empresas no ramo e produzindo 48\% de empregos na Amazônia (PAIVA, 2009). Possuindo um destaque socioeconômico, o setor florestal utilizando 300 milhões de $\mathrm{m}^{3}$ de madeira por ano gera em torno de dois milhões de empregos detendo 2 bilhões de impostos anuais (NOCE et al., 2005). O beneficiamento da madeira extraída no Estado do Pará é feito por pequenas e 
microempresas de madeira serradas, móveis e artefatos de madeira formando concentração industrial devido a diversificação de produtos provenientes da extração da madeira em tora (SANTANA, 2002).

O consumo da madeira coloca o Estado do Pará como um dos maiores exportadores de madeira sendo $64 \%$ designados para o mercado doméstico e $36 \%$ para a exportação, pelo país o Sul e Sudeste com 27\% são as regiões com o maior consumo de madeira da região amazônica, seguido por São Paulo e a Amazônia Legal com 11\% e o Nordeste com 7\% (SANTOS, 2009).

No Pará os municípios de Belém, Ananindeua e Paragominas são considerados os maiores exportadores de madeira tendo Paragominas 39\% das exportações, no entanto apesar do destaque no setor florestal, o mesmo se encontra em crise devido à escassez de matériaprima pela insciência científica de espécies da Amazônia (REIS, 2015).

Em 2009 o setor madeireiro foi atingido pela crise internacional o que afetou as exportações de madeira da Amazônia. No Pará, por exemplo, a Associação das Indústrias Exportadoras de Madeiras do Estado (Aimex) comparou os dados de exportação estadual entre 2007 e 2009 e registrou uma queda relevante. Em 2007, o valor exportado foi US\$ 793 milhões, contra US\$ 631 milhões em 2008 e US\$ 346 milhões em 2009 (AIMEX, 2010). Ademais, o impacto da crise econômica foi bem menor na economia brasileira. Considerando que historicamente, a grande maioria da madeira produzida na Amazônia destina-se ao mercado nacional (SOBRAL et al. 2002; LENTINI et al., 2003; LENTINI et al. 2005), a crise econômica teve um impacto moderado na queda da produção madeireira amazônica, porém considerável para a economia do estado na época.

E, conforme a Secretaria de Estado e Meio Ambiente do Estado do Pará (SEMA-PA) houve aumento no consumo de madeira em tora a partir de 2009 no Estado do Pará, apresentando valores de 2,7 milhões de metros cúbicos em (2009), 3,7 milhões de $\mathrm{m}^{3}$ (2010) e 4,2 milhões de $\mathrm{m}^{3}$ em (2011), respectivamente.

Em acordo com o estudo do Imazon (SOUZA Jr. et al. 2000), a extensa rede de estradas, o relevo suavemente ondulado e as boas condições de navegabilidade dos rios do Pará fazem com que a maioria $(77 \%)$ das florestas do Estado sejam economicamente acessíveis à atividade madeireira. Por outro lado, aproximadamente $23 \%$ são florestas consideradas inacessíveis por estarem situadas em áreas de relevo acidentado, rios não-navegáveis e uma precária rede de estradas. Essas florestas remotas estão localizadas principalmente no extremo norte do Estado. (VERÍSSIMO, A.; LIMA, E.; LENTINI, M. 2002)

\section{METODOLOGIA}


A área de estudo é a mesorregião do Sudoeste Paraense (Figura 1) apresentando uma extensão territorial de $415789 \mathrm{~km}^{2}$, composta por quatorze municípios: Aveiro, Jacareacanga, Rurópolis, Pacajá, Altamira, Novo Progresso, Itaituba, Anapu, Brasil Novo, Medicilândia, Trairão, Senador José Porfirio, Uruará, Vitória do Xingu. Os dados utilizados foram obtidos por meio dos relatórios de Extração e Comércio de Toras de Madeira Nativa por Município e, Beneficiamento e Comércio de Produtos de Madeira por Espécies Florestais da Secretária de Estado de Meio Ambiente e Sustentabilidade - SEMAS, obtidos pelo Sistema de Comercialização e Transporte de Produtos Florestais - SISFLORA. Os relatórios possuem o nome científico, quantidade de Guias Florestais (GF), o volume em metro cúbico e valor comercializado das espécies.

Figura 1: Localização da mesorregião Sudoeste Paraense no Estado do Pará.

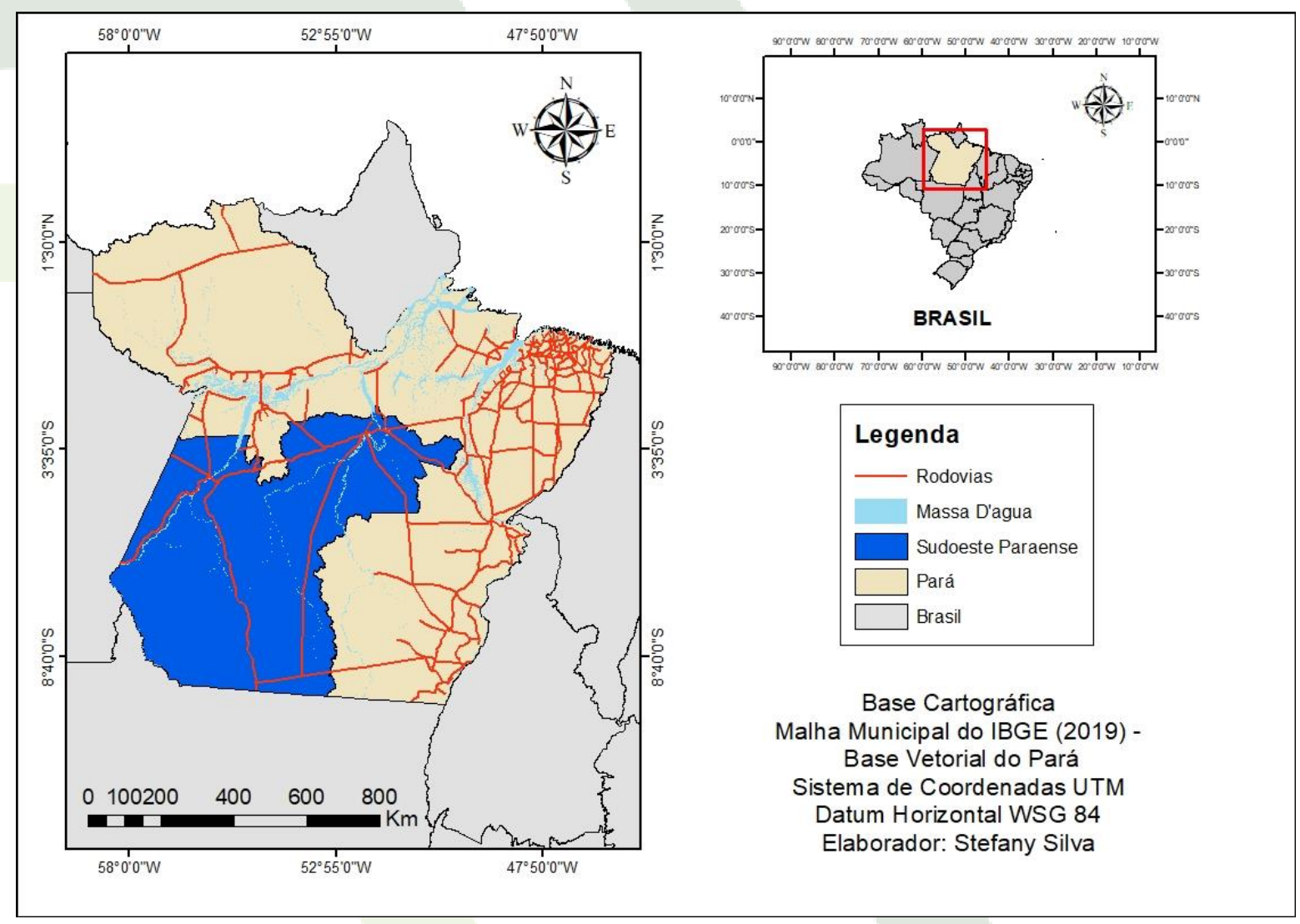

Fonte: Própria, 2020

Os dados de Extração e Comércio de Toras de Madeira Nativa por Município foram transferidos para o Excel a fim de gerar gráficos e tabelas da espécie mais explorada, produção $\left(\mathrm{m}^{3}\right)$ e valor da produção (mil reais), nos quatorze municípios do Sudoeste do Pará. Em seguida os dados de Beneficiamento e Comércio de Produtos de Madeira por Espécies Florestais, serão deslocados para o Excel para analisar o beneficiamento, volume $\left(\mathrm{m}^{3}\right)$, o valor comercializado e 
o valor médio das espécies mais exploradas no Sudoeste Paraense no período de 2006 até 2016

\section{RESULTADOS E DISCUSSÃO}

Foram analisados os dados de 14 municípios que fazem parte do Sudoeste Paraense, com base no relatório de Extração e Movimentação de Toras de Madeira Nativa por município da Secretária de Estado do Meio Ambiente (SEMA), a partir disso ocorreu a extração de dados da espécie de maior receita, volume e o beneficiamento dessas espécies madeireiras.

A espécie conhecida vulgarmente como "Ipê" foi a que teve o maior valor de comercialização por volume em $\mathrm{m}^{3}$ no período consolidado de janeiro de 2006 à fevereiro de 2016 nos seguintes locais Rurópolis, Trairão, Uruará, Senador José Porfírio, Itaituba, Altamira e Anapu foram os pioneiros como é mostrado no gráfico 01.

Gráfico 01: Valores do "Ipê" nos municípios.

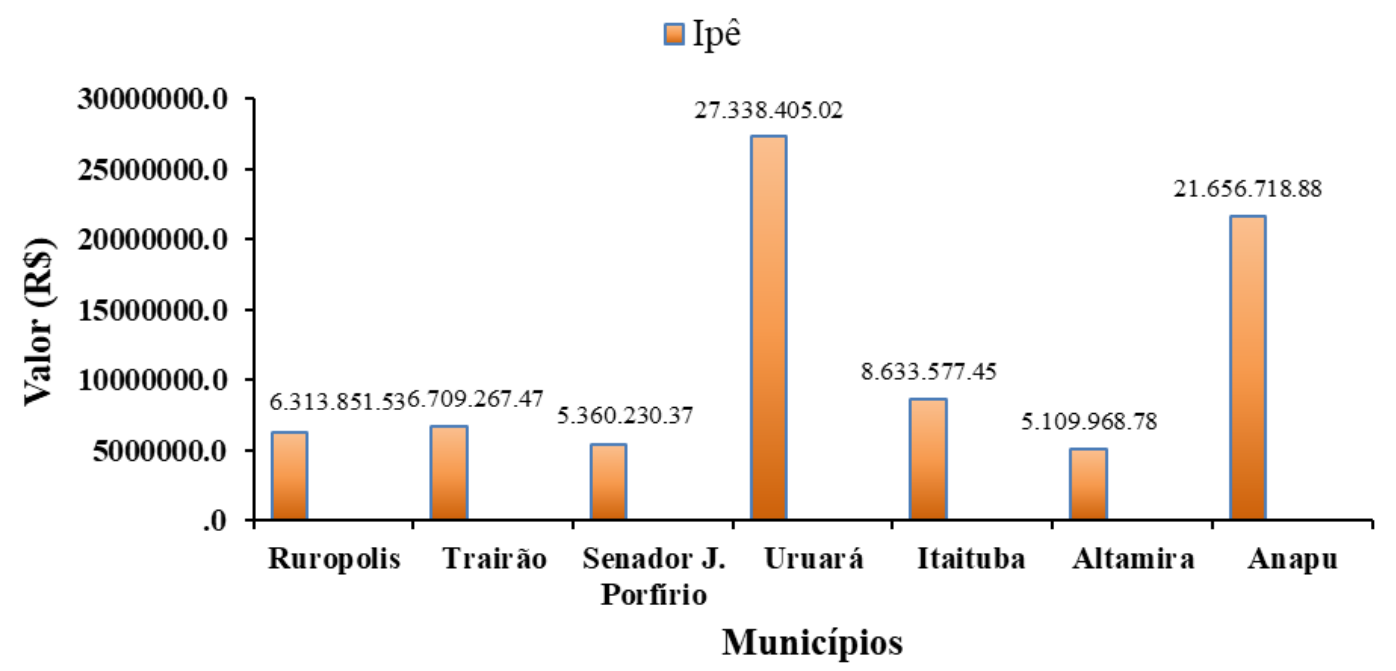

Fonte: Autores (2020)

Nesses setes municípios as espécies madeireiras de nomes científicos atribuídos ao ipê foram doze, que são: Macrolobium pendulum Willd. Ex Vogel, Tabebuia barbata (E.Mey.) Sandwith, Tabebuia capitata (Bureau \& K.Schum.) Sandwith, Tabebuia serratifolia (Vahl) Nichols, Tabebuia cassinoides Lam.) DC, Tabebuia ochracea (Cham.) Standl, Tabebuia chrysotricha (Mart. ex DC.) Standl., Tabebuia heptaphylla (Vell.) Toledo, Tabebuia rígida Dutra, Tabebuia impetiginosa (Mart. Ex DC.) Standl, Tabebuia serratifolia, Tabebuia umbellata (Sond.) Sandwith.

E cada uma delas foi analisado quatro tipos de produto do beneficiamento das espécies que são: aproveitamentos de madeira, decking, madeira serrada e toras de madeira nativa e 
como resultado foi alcançado que a espécie Tabebuia serratifolia (Vahl) Nichols teve o maior valor em vol.m³ (tabela 01$)$.

Tabela 01: Análise dos produtos de beneficiamento da espécie madeireira Tabebuia serratifolia (Vahl) Nichols.

\begin{tabular}{|c|c|c|c|c|}
\hline Produto & Unidade & Volume & Valor $(\mathrm{R} \$)$ & Valor médio \\
\hline $\begin{array}{l}\text { Aproveitamentos } \\
\text { de madeira }\end{array}$ & $\mathrm{m}^{3}$ & 7.848, 0741 & 6.053 & 771,34 \\
\hline Madeira serrada & $\mathrm{m}^{3}$ & $271.615,4861$ & $438.646 .975,39$ & $1.614,95$ \\
\hline Decking & $\mathrm{m}^{3}$ & $540.169,0406$ & $1.790 .330 .895,90$ & $1.826,05$ \\
\hline $\begin{array}{l}\text { Toras de madeira } \\
\text { nativa }\end{array}$ & $\mathrm{m}^{3}$ & $177.796,5427$ & $75.623 .788,42$ & 425,33 \\
\hline Total & & $9997.429,142$ & $\mathrm{R} \$ 2.310 .655 .236,27$ & $4.637,67$ \\
\hline
\end{tabular}

Fonte: Autores (2020)

As madeiras do ipê têm sido utilizadas na construção civil em geral, marcenaria, tábuas de assoalho, decking, postes, pilares, estruturas externas, pontes (LORENZI, 2008; PIOVESAN et al., 2011). Por serem pesadas, duras, de coloração pardo-acastanhada, com seus vasos obstruídos por ipeína (substância de cor amarela-esverdeada). Essas características explicam o motivo dela ser tão extraída e ter valor de comercialização sendo um dos maiores do estado do Pará.

A segunda espécie por nome vulgar com maior receita por volume $\mathrm{em}^{3}$ foi a Maçaranduba que teve os maiores valores no município de Brasil Novo, Aveiro e Pacajá. Na tabela 02 é possível ter os dados de valor e volume $\mathrm{em}^{3}$ de cada município.

Tabela 02: Valor e volume da "maçaranduba" nos municípios.

\begin{tabular}{|lccc|}
\hline & Município & Valor (R\$) & Volume $\left(\mathrm{m}^{3}\right)$ \\
\hline & & \\
Brasil Novo & $1.699 .487,62$ & $10.896,7613$ \\
Aveiro & $18.564 .179,41$ & 89350,9504 \\
Pacajá & $20.582 .432,37$ & 131015,7944 \\
\hline \multicolumn{2}{|c|}{} & $40.846 .099,3$ & $221.456,506$ \\
\hline
\end{tabular}

Fonte: Autores (2020)

A partir dos dados do seu beneficiamento (Tabela 03) foi constatado que a espécie madeireira Manilkara huberi (Ducke) Chevalier teve resultados de maior valor com os quatros tipos de produtos apresentados. Apesar de que o valor não ultrapassou o do Ipê, que foi considerado em segundo lugar no ranking. 
Tabela 03: Beneficiamento dos produtos da espécie madeireira Manilkara huberi (Ducke) Chevalier

\begin{tabular}{|c|c|c|c|c|}
\hline Produto & Unidade & Volume & Valor $(\mathrm{R} \$)$ & Valor médio \\
\hline Aproveitamento da madeira & $\mathrm{m}^{3}$ & $50.512,74$ & $8.871 .137,19$ & 175,62 \\
\hline Madeira Serrada & $\mathrm{m}^{3}$ & $251.335,52$ & $138.897 .297,60$ & 552,63 \\
\hline Decking & $\mathrm{m}^{3}$ & $178.638,00$ & $380.295 .241,27$ & $2.128,85$ \\
\hline Toras de madeira nativa & $\mathrm{m}^{3}$ & $1.092 .690,94$ & $181.800 .663,78$ & 166,37 \\
\hline Total & & $1.573 .177,19$ & $709.864 .339,84$ & 3023,47 \\
\hline
\end{tabular}

Fonte: Própria (2020)

Essa espécie madeireira pode ser considerada de alta densidade, bem como vale citar sua resistência física à ação do tempo além da resistência biológica a fungos e cupins sendo, por muitas vezes, utilizada em contato direto com o solo na forma de vigas, tábuas e tacos para assoalho (CARVALHO, 2007; \& PEREIRA et al., 2011).

O município que teve o menor valor de extração e comercialização de madeira foi Jacareacanga, e a espécie com maior volume e valor foi o Tauari com quatro nomes científicos associados ao seu nome vulgar como pode ser visto na tabela 04 .

Tabela 04: Espécie mais explorada no municipio de Jacareacanga.

\begin{tabular}{|c|c|c|}
\hline Espécie & Valor (R\$) & Volume em $\mathrm{m}^{3}$ \\
\hline Tauari - Couratari guianensis Aubl. & $389.010,37$ & 8606,715 \\
\hline \multicolumn{3}{|c|}{ Tauari - Couratari oblongifolia Ducke \& } \\
\hline R.Knuth & $102.858,03$ & 2253,94 \\
\hline Tauari - Couratari stellata A.C.Sm. & $130.044,57$ & 2836,059 \\
\hline Tauari - Couratari tauari O.Berg & $122.101,30$ & 1471,1 \\
\hline Total Geral & $744.014,27$ & 15167,814 \\
\hline
\end{tabular}

Fonte: Própria (2020)

Mesmo com a menor receita e volume total de extração e comercialização, foi analisado os dados de beneficiamento e foi constatado como é exposto na tabela 05 que a espécie madeireira Couratari guianensis Aubl foi a que destacou e como maior aproveitamento "toras de madeira nativa" apesar do município não ser tão referente.

Tabela 05: Beneficiamento da espécie Couratari guianensis Aubl

\begin{tabular}{|l|c|c|c|c|}
\hline Produto & Unidade & Volume & Valor $(\mathrm{R} \$)$ & Valor médio \\
\hline Aproveitamento da madeira & $\mathrm{m}^{3}$ & $7.360,45$ & $2.875 .307,69$ & 390,64 \\
Madeira Serrada & $\mathrm{m}^{3}$ & $61.560,15$ & $42.540 .710,42$ & 691,04 \\
Decking & $\mathrm{m}^{3}$ & 868,8999 & $1.614 .525,76$ & $1.858,12$ \\
Toras de madeira nativa & $\mathrm{m}^{3}$ & $80.534,38$ & $12.484 .997,06$ & 155,02 \\
\hline \multicolumn{2}{|c|}{ Total } & $150.323,88$ & $59.515 .540,93$ & 3094,82 \\
\hline
\end{tabular}


Fonte: Própria (2020)

O município de Uruará foi o pioneiro na extração e comercialização somando ao total $\mathrm{R} \$ 27.338 .405,02$ como é mostrado no gráfico 02 o ranking dos valores apenas da sua espécie de maior valor e volumetria foi o Ipê, o mais explorado do Sudoeste Paraense.

Gráfico 02: Ranking dos municípios da espécie mais explorada e com maior valor.

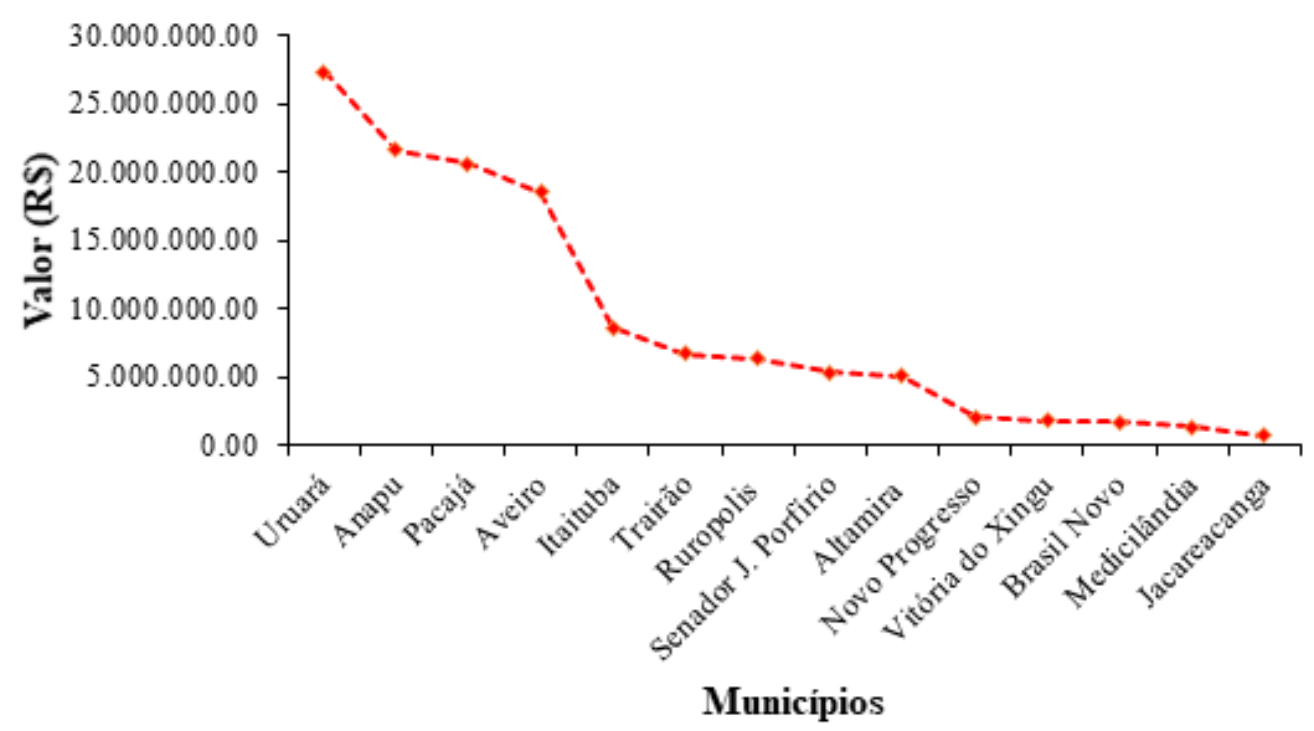

Fonte: Própria (2020)

Os municipios da mesorregião Sudoeste Paraense, apresentaram uma produção total de 1.657.410,11 m3 no qual o municipio de Pacajá teve o maior valor como é exposto na figura 01. A receita gerada foi de $\mathrm{R} \$ 230.522 .780$ (Figura 02) contribuindo para a economia da região, liderando na extração e comércio de toras de madeiras nativas no período de 2006 à 2016.

Figura 01: Produção por volume $\mathrm{m}^{3}$. 


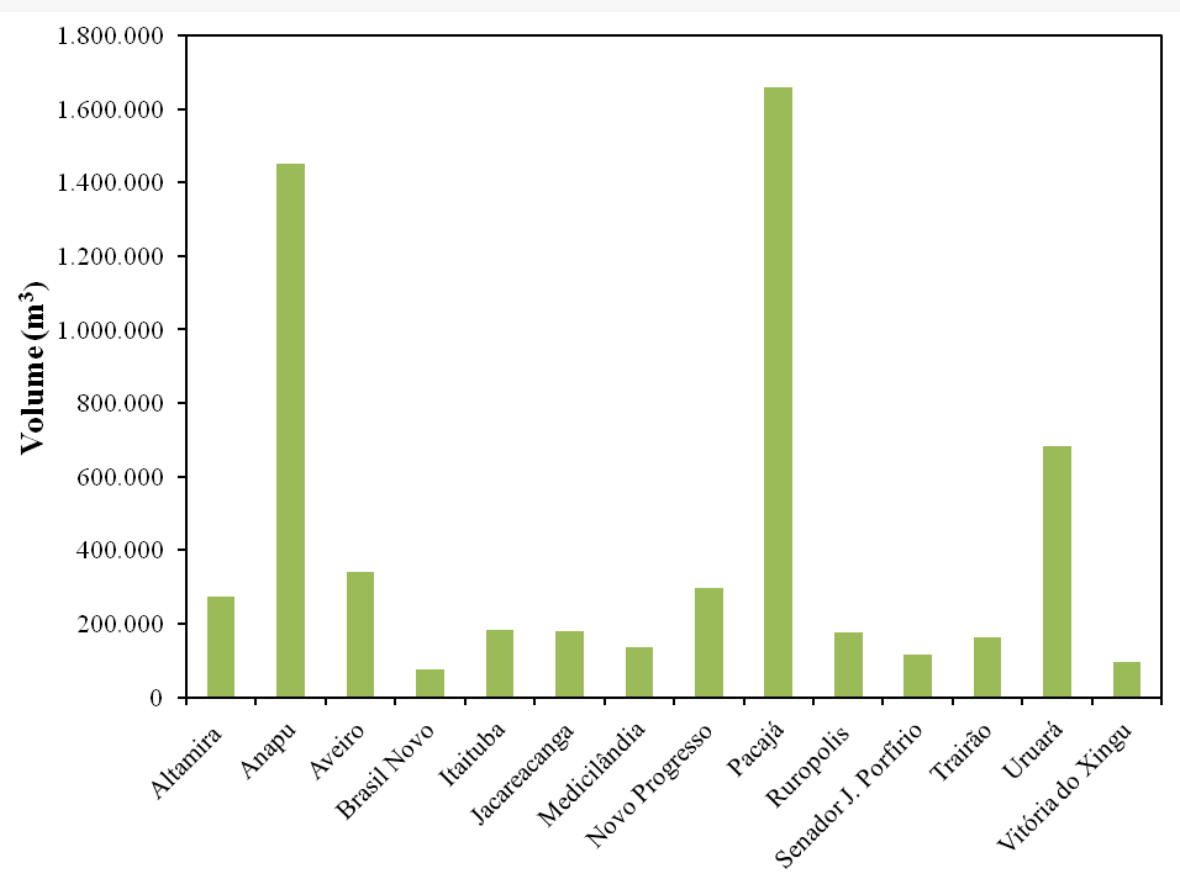

Fonte: Própria (2020)

Figura 02: Receita dos municipios do Sudoeste Paraense.

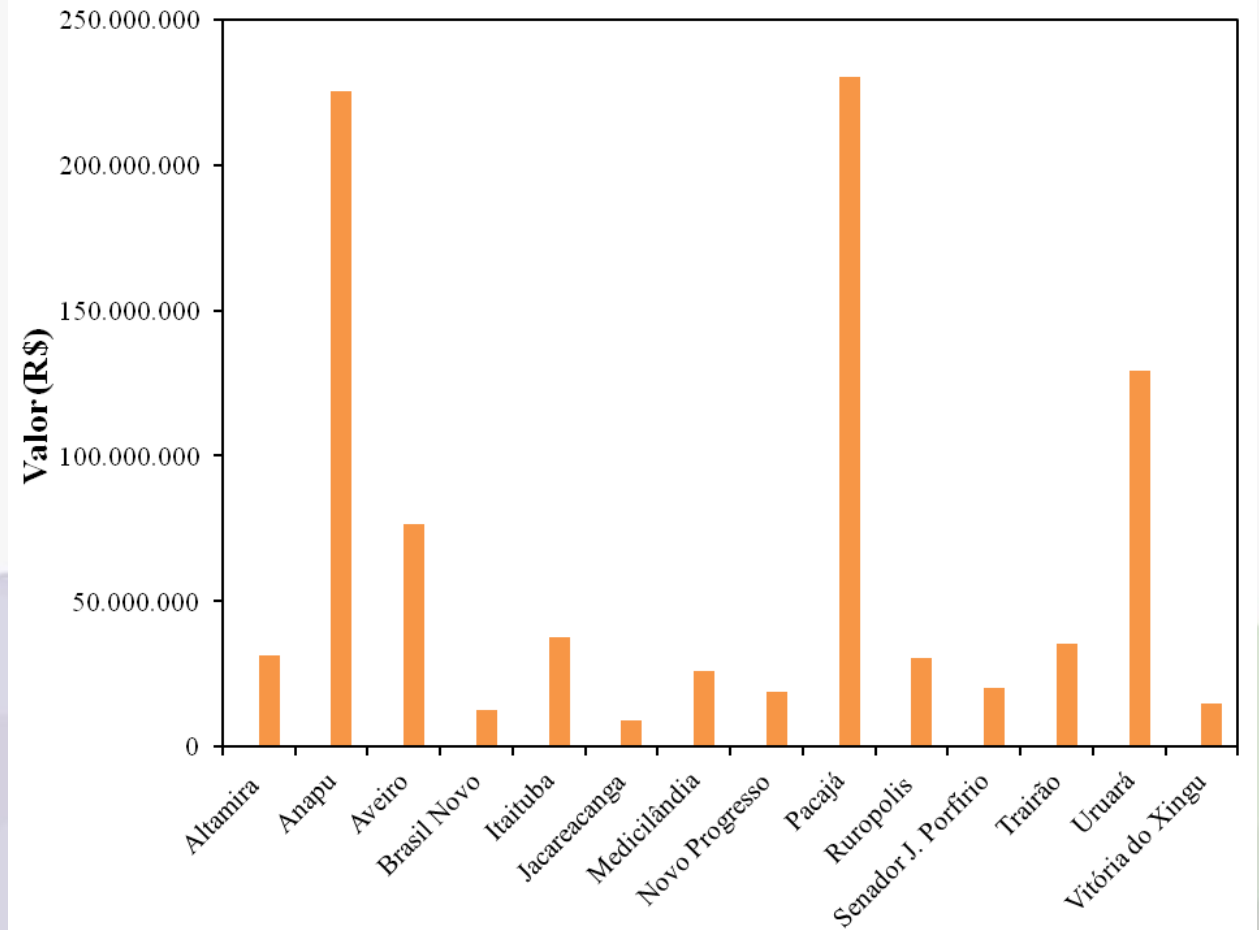

Fonte: Própria (2020)

\section{CONCLUSÕES}

Os resultados apresentam o Ipê com a maior comercialização de madeira por volume em $\mathrm{m}^{3}$ em sete municípios do sudoeste paraense: Rurópolis, Trairão, Uruará, Senador José Porfírio, Itaituba, Altamira e Anapu, sendo Uruará o município com a maior receita em 
R\$27.338.405,02, seguido de Anapu com R\$21.656.718,88. Já para o beneficiamento, o Ipê Tabebuia serratifolia (Vahl) Nichols teve o maior valor em vol. $\mathrm{m}^{3}$ entre os produtos de madeira serrada, toras de madeira nativa, decking e aproveitamento de madeira totalizando $9997.429,142 \mathrm{~m}^{3}$ e receita de $\mathrm{R} \$ 2.310 .655 .236,27$, tendo destaque para os produtos de decking e madeira serrada.

A Maçaranduba apresentou os maiores valores nos municípios de Brasil Novo, Aveiro e Pacajá, totalizando 40.846.099,3 em volume por $\mathrm{m}^{3}$ e a receita em $\mathrm{R} \$ 221.456,506$. Os dados para o beneficiamento totalizaram o volume $1.573 .177,19 \mathrm{~m}^{3}$ e a receita em $\mathrm{R} \$ 709.8664 .339,84$, destacando os produtos de madeira serrada e toras de madeira nativa. $\mathrm{O}$ município do sudoeste paraense com menor valor de extração e comercialização foi Jacareacanga para o Tauari totalizando o volume de $15167,814 \mathrm{~m}^{3}$ e a receita de $\mathrm{R} \$ 744.014,27$, para o beneficiamento foi totalizado um volume de $150.323,88 \mathrm{~m}^{3}$ e a receita em $\mathrm{R} \$ 59.515 .540,93$. A soma total dos municipios apontam que Pacajá gerou $\mathrm{R} \$ 230.522 .780,47$ e totalizando o volume de 1.657.410,11 de extração e comércio de madeira em tora.

Sendo assim, esse estudo permite analisar o setor florestal de madeiras em tora das mesorregiões do Estado patenteando a economia desse mercado em escala nacional e internacional sempre respeitando os princípios da sustentabilidade.

\section{REFERÊNCIAS}

ASNER, G. P., KELLER, M., LENTINI, M., MERRY, F., SOUZA JUNIOR, C. Extração seletiva de madeira e sua relação com o desmatamento. Amazonia and Global Change Geophysical Monography, 2009a, 17 p.

BRASIL. Ministério do Meio Ambiente (MMA). Biomas: Amazônia. 2016. Disponível em: . Acesso em: 20 de setembro de 2020.

CARVALHO, J. Utilização da madeira na construção civil. Centro de apoio ao desenvolvimento tecnológico da Universidade de Brasília - CDT/UnB, 2007.

DE SANTANA, A. C. Análise da competitividade sistêmica da indústria de madeira no Estado do Pará. Revista de Economia e Agronegócio, v. 1, n. 2, 2003.

DING, Y., ZANG, R., LU, X., HUANG, J. The impacts of selective logging and clearcutting on woody plant diversity after 40 years of natural recovery in a tropical montane rain forest, south China. Science of the Total Environment, v. 579, p. 1683-1691, 2017.

HUMMEL, A. C.; ALVES, M. D. S.; PEREIRA, D.; VERÍSSIMO, A.; SANTOS, D. A atividade madeireira na Amazônia brasileira: Belém, PA: Serviço Florestal Brasileiro (SFB); Instituto do Homem e Meio Ambiente da Amazônia- Imazon, 2010.

LENTINI, M.; VERÍSSIMO, A.; SOBRAL, L. Fatos florestais da Amazônia 2003. Belém: Imazon. 110 p.2003. 
LENTINI, M.; PEREIRA, D.; CELENTANO, D.; PEREIRA, R. 2005. Fatos florestais da Amazônia. Belém: Imazon. 141 p.2005.

LENTINI, M.; VERÍSSIMO, A.; PEREIRA, D. A expansão madeireira na Amazônia. Revista da madeira-edição $\mathbf{N}^{\circ} \mathbf{9 8}, 2006$.

LORENZI, H. Árvores Brasileiras: manual de identificação de plantas arbóreas do Brasil. Nova Odessa, SP: Instituto Plantarum v. 1, ed. 5.p., 2008.

LUZ, E. S. Mercado de madeira e qualidade de toras de espécies tropicais no estado do Pará: subsídios para o manejo florestal sustentável, 2015.

MELO, JE de; CORADIN, V. T. R.; MENDES, J. C. Classes de densidade para madeiras da Amazônia brasileira. In: Anais do Congresso Florestal Brasileiro. São Paulo: Sociedade Brasileira de Silvicultura, 1990. p. 695-699

NOCE, R.; SILVA, M. L. D.; CARVALHO, R. M. M. A.; SOARES, T. S. Concentração das exportações no mercado internacional de madeira serrada. Revista Árvore, v. 29, n. 3, p. 431 437, 2005.

NUNES, P. A.; MELO, C. O.; TEIXEIRA, D. A participação do setor madeireiro na economia das microrregiões geográficas do Paraná — 2009. Revista Brasileira de Agropecuária Sustentável, v. 2, n. 1, p. 8-20, jul. 2012.

PAIVA CÁtiA, V. B. Análise da Produção Madeireira no Estado do Pará. Monografia (Título de Engenheiro Florestal) - Universidade Federal Rural do Rio de Janeiro, Rio de Janeiro.2009.

PEREIRA, L. C. O; TAKAHASHI, R.; FILHO, R. V. V.; OLIVEIRA, D. R.C.; FUJIYAMA, R. T. Caracterização de resíduo de madeira e fibras de sisal para fabricação de materiais compósitos de matriz poliéster. In: CONGRESSO BRASILEIRO DE EDUCAÇÃO E ENGENHARIA, 39, Blumenau.2011.

PIOVESAN, P. R. R.; SOUSA, A. S.; REIS, A. R. S. Caracterização do setor madeireiro no município de Uruará, Pará, Brasil. In: Seminário de Educação, Ciência e Tecnologia do IFPA no Xingu, Altamira. Anais...IFPA: Altamira, 2011.

REIS, A. R. S. Anatomia da madeira de quatro espécies de Aspidosperma Mart. \& Zucc. Comercializadas no estado do Pará, Brasil. Revista Ciência da Madeira (Brazilian Journal of Wood Science), v. 6, n. 1, p. 10-12953/2177-6830/rcm. v6n1p47-62, 2015.

SANTOS, R. B. N.; SANTANA, A. C. Comportamento recente do setor florestal madeireiro no estado do Pará, Brasil. Revista Árvore, v. 33, n. 3, p. 533-543, 2009.

SOBRAL, L.; VERÍSSIMO, T.; LIMA, E.; AZEVEDO, T.; SMERALDI, R. Acertando o alvo 2: consumo de madeira amazônica e certificação florestal no Estado de São Paulo. Belém: Imazon, Imaflora e Amigos da Terra. 74 p.2002.

SERVIÇO FLORESTAL BRASILEIRO - SFB/ INSTITUTO DO HOMEM E MEIO AMBIENTE DA AMAZÔNIA - IMAZON. A atividade madeireira na Amazônia brasileira: produção, receita e mercados. Belém, PA: Serviço Florestal Brasileiro (SFB); Instituto do Homem e Meio Ambiente da Amazônia (IMAZON), 2010 
AVALIAÇÃo DA EXTRAÇÃO, COMÉRCIO E BENEFICIAMENTO DE MADEIRA

VERÍSSIMO, A; L. E; L. M. Pólos Madeireiros do Estado do Pará. Belém, Imazon. 74 p.2002.

VERÍSSIMO, A.; BARRETO, P.; MATTOS, M.; TARIFA, R.; UHL, C. Logging impactos and prospects for sustainable forest management in an old Amazon frontier: the case of Paragominas. Forest Ecology and Management, v. 55, p. 169-199. 1992. 